\title{
Rituximab Synergizes with Hydroxyurea or Vincristine in the Killing of Ramos Burkitt's Lymphoma B Cell Line
}

\author{
Mohamed Deyab, Abdulrhman Elbanani, ${ }^{1}$ Salah Tabal, ${ }_{1}^{1}$ Hajer Geriani, ${ }^{1}$ Yosra Lamami, \\ Amin Bredan, ${ }^{2,3}$ and Abdulmunem Abulayha ${ }^{1}$
}

\begin{abstract}
Rituximab is an effective immunotherapy for CD20-positive B-cell non-Hodgkin's lymphoma. However, some patients show resistance, particularly those suffering from more aggressive lymphoma types, such as Burkitt's lymphoma. Hence, Rituximab is commonly combined with several chemotherapeutic drugs. With a view to reduce the number of such drugs, we examined the effect of combining Rituximab individually with hydroxyurea, vincristine, or etoposide on the killing of Ramos Burkitt lymphoma cell line type I. Cell death was examined by using Annexin-V/propidium iodide staining. Combining Rituximab with hydroxyurea or vincristine resulted in a synergistic effect, whereas combining it with etoposide resulted in a subadditive effect. In single treatments, the percentage of cell death ranged from 23\% (Rituximab) to 36\% (hydroxyurea). Combining Rituximab with hydroxyurea or vincristine resulted in a synergistic effect ( $83 \%$ and $74 \%$ killing, respectively). In contrast, only a subadditive effect was noticed with etoposide (36\%). We conclude that the synergistic effect of Rituximab with hydroxyurea or vincristine is worthy of further study, and that further in vitro screening of chemotherapeutics might identify chemo-immunotherapeutic combinations that are effective in vivo but less toxic than currently used regimens.
\end{abstract}

Key words: chemotherapy, etoposide, hydroxyurea, Rituximab, vincristine

\section{Introduction}

$\mathbf{R}$ ituximab is highly effective in treating a wide range of lymphoproliferative disorders, including many B-cell lymphomas (reviewed in Plosker and Figgitt ${ }^{1}$ ). Nevertheless, some patients with indolent follicular B cell lymphoma relapse repeatedly, and the response rate is still lower in patients with more aggressive types of B-cell non-Hodgkin's lymphoma (NHL). ${ }^{2,3}$ Rituximab is also combined with chemotherapy to improve long-term outcome. One of the most commonly used immune-chemotherapeutic combinations is R-CHOP, which combines Rituximab with cyclophosphamide, doxorubicin, vincristine, and prednisone. ${ }^{4} \mathrm{R}-\mathrm{CHOP}$ shows a $95 \%$ response rate in indolent lymphomas ${ }^{5}$ and $94 \%$ in aggressive lymphomas. ${ }^{6}$ However, some types of lymphoma, such as Burkitt's lymphoma, require even more intense chemotherapeutic treatment.
Although the introduction of chemo-immunotherapeutic combinations has improved the outcome of NHL patients, some individuals do not respond to treatment and relapses remain common. Therefore, new effective strategies for NHL therapy are needed. The strategies should aim at using as few chemotherapeutic agents with Rituximab as possible to reduce cytotoxicity while inducing a highly effective synergistic effect. We compared the effects of separately combining three different antitumor compounds with Rituximab on the cell death of a Burkitt's lymphoma cell line. These compounds are commonly used in therapy and research and have different mechanisms of action: hydroxyurea, a competitive inhibitor of ribonucleotide reductase ${ }^{7}$; vincristine, a vinca alkaloid that inhibits the formation of mitotic spindles ${ }^{8}$; and etoposide, a potent inhibitor of topoisomerase II. ${ }^{9} \mathrm{We}$ intended to observe whether the chemotherapeutics would act synergistically or additively with Rituximab.

\footnotetext{
${ }^{1}$ Cell Biology Research Group, Biotechnology Research Center, Twisha, Tripoli, Libya.

${ }^{2}$ Inflammation Research Center, VIB, Ghent, Belgium.

${ }^{3}$ Department of Biomedical Molecular Biology, Ghent University, Ghent, Belgium.
}

Address correspondence to: Abdulmunem Abulayha; Cell Biology Research Group, Biotechnology Research Center; Sedra Road, Twisha, P.O. Box 30313, Tripoli, Libya

E-mail: abdulmunem09@hotmail.com 


\section{Materials and Methods}

Antibodies and reagents

Rituximab (MabThera; Roche Diagnostics; stock solution $10 \mathrm{mg} / \mathrm{mL}$ ) was a kind gift from the National Oncology Institute (Sabratah, Libya). Unless stated otherwise, all other reagents were purchased from Sigma Chemical Company.

\section{Cell lines}

CD20-expressing human Burkitt's lymphoma cell line (Ramos) was purchased from the European Collection of Cell Culture (ECACC) (Wiltshire SP4 0JK, UK) and maintained in RPMI-1640 medium supplemented with 10\% heatinactivated fetal bovine serum, $200 \mathrm{mM}$ L-glutamine solution that was sterile filtered and cell culture tested, and penicillinstreptomycin that was sterile filtered and cell culture tested. The cells were incubated at $37^{\circ} \mathrm{C}$ in $5 \% \mathrm{CO}_{2}$. Viability was estimated by trypan blue exclusion, and cells were used only if their viability exceeded $90 \%$.

\section{Cell treatments and Annexin-V-based cell death analysis}

Cells were cultured at $1 \times 10^{5}$ cells $/ \mathrm{mL}$ in $25-\mathrm{cm}^{2}$ tissue culture plates and treated with $10 \mu \mathrm{g} / \mathrm{mL}$ Rituximab, either alone or in combination with $1 \mathrm{mM}$ hydroxyurea, $2 \mu \mathrm{M}$ vincristine, or $10 \mu \mathrm{M}$ etoposide for 24 or 48 hours. They were then washed in phosphate-buffered saline and resuspended in binding buffer containing $0.25 \mu \mathrm{g} / \mathrm{mL}$ Annexin-V and $5 \mu \mathrm{g} /$ $\mathrm{mL}$ propidium iodide (PI; BD Biosciences) and incubated in the dark for $15 \mathrm{~min}$ at room temperature before analysis. Single-cell suspensions were prepared for cytometric analysis.

\section{Flow cytometry analysis}

Calibration, parameter optimization, and compensation for the overlap between the different fluorochrome spectra
FIG. 1. Representative of flow cytometry plots of Ramos cell death induced by rituximab or chemotherapy agents; FL1 (Annexin$\mathrm{V})$ versus FL2 (PI) after $48 \mathrm{~h}$ of treatment. Rituximab $10 \mu \mathrm{g} / \mathrm{mL}$; hydroxyurea $1 \mathrm{mM}$; vincristine $2 \mu \mathrm{M}$; etoposide $10 \mu \mathrm{M}$. Annexin$\mathrm{V}^{+\mathrm{ve}} / \mathrm{PI}^{-\mathrm{ve}}$ (early apoptotic cells) are those in the lower right quadrant, whereas Annexin- $\mathrm{V}^{+\mathrm{ve}} / \mathrm{PI}^{+\mathrm{ve}}$ (late apoptotic/necrotic cells) are in the upper right quadrant.

\section{A}

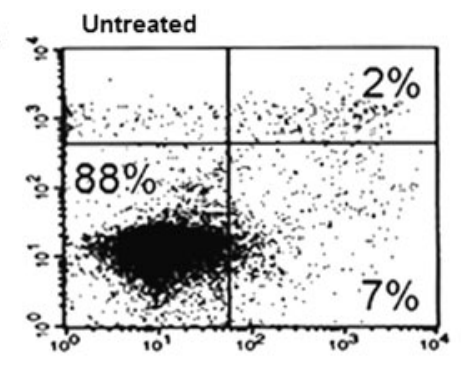

c

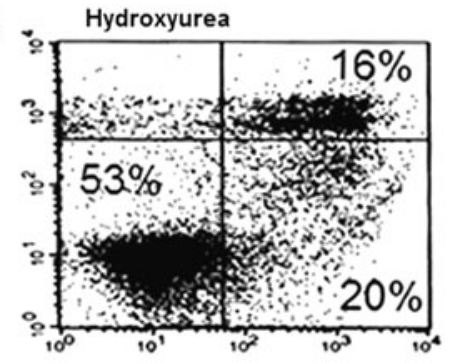

E

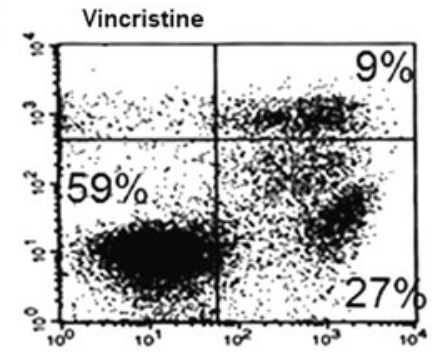

G.

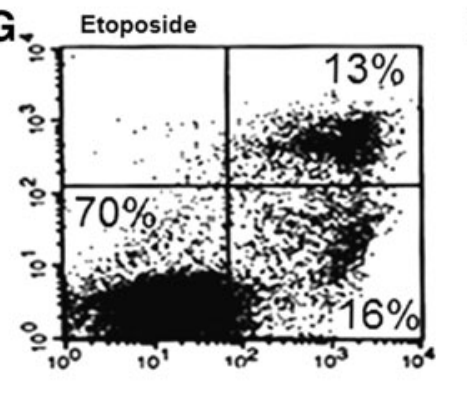

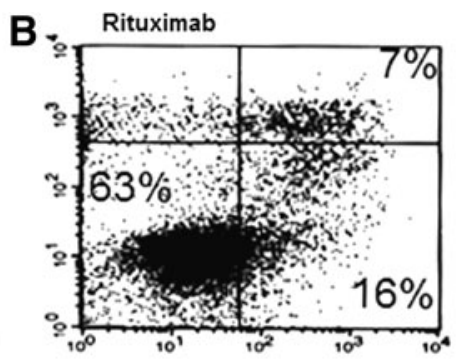

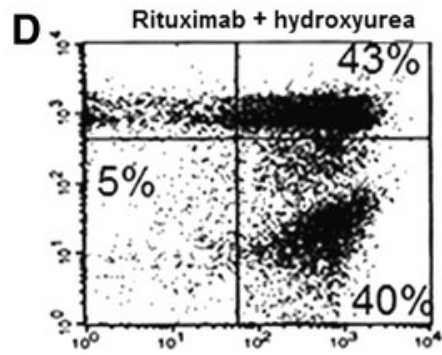

$F$
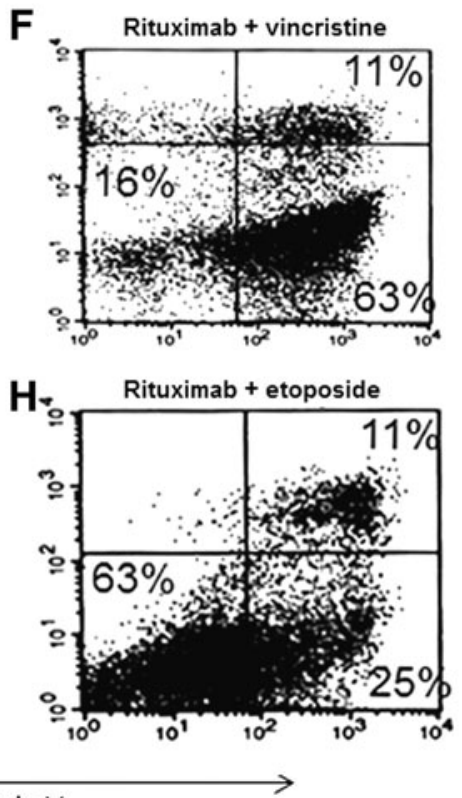
were obtained automatically using FacsComp software (Becton Dickinson). Analysis and calculations were performed using CellQuest (Becton Dickinson). A minimum of 20,000 events were collected.

\section{Statistical analysis}

All values are given as the mean \pm standard deviation (SD). One-sample $t$-test was used to compare the effect of combined treatment with the mathematical sum of the corresponding individual treatments.

\section{Results}

Three chemotherapeutics were evaluated for their ability to synergize with Rituximab in the induction of cell death in the Ramos B-cell line. For all treatments, as the time of incubation increased ( 24 and 48 hours) more cells became positive for both Annexin-V and PI (results not shown). Figure 1 shows representative plots of flow cytometric analysis of cell death.

Percentages of total apoptosis after 48 hours of incubation are shown in Figure 2. Rituximab alone induced $23.5 \% \pm 0.74 \%$ (SD) apoptosis. Etoposide alone induced $29.8 \% \pm 0.61 \%$, vincristine $34.6 \% \pm 1.92 \%$, and hydroxyurea $36.1 \% \pm 0.35 \%$. The effect of the Rituximab-etoposide combination was subadditive $(36.2 \% \pm 0.52 \%)$. However, hydroxyurea and vincristine synergized significantly with Rituximab to induce $83.0 \% \pm 0.49 \%$ and $74.4 \% \pm 0.10 \%$ apoptosis, respectively (both $P<0.001$ compared to mathematical sum of individual treatments).

\section{Discussion}

In the treatment of B-cell NHLs, Rituximab alone induces apoptosis only in a subpopulation of cells, whereas the rest continue to proliferate. According to the literature, Rituximab is effective against indolent NHL but not against aggressive types. ${ }^{3}$ For that reason, standard chemotherapeutic agents are used to increase the efficacy of treatment. One of the most widely used combinations is R-CHOP, in which Rituximab is combined with cyclophosphamide, doxorubicin, vincristine, and prednisone. But in the absence of convincing in vitro evidence, most clinical trials have often taken an experimental approach with a broad range of combinations. Systematic in vitro studies might identify simpler immune-chemotherapeutic combinations that are as effective as current combinations but are less toxic.

Burkitt's lymphoma is highly aggressive, and up to eight different chemotherapeutic drugs, alone or with Rituximab, may be used in its treatment. The Ramos cell line was derived from a human Burkitt's lymphoma and is hence representative of this type of neoplasm. In vitro, monomeric Rituximab induces only modest cell death in Ramos cells. ${ }^{10,11}$ In vivo, a recent retrospective study reported that the intensive chemotherapy regimens used for adult Burkitt's lymphoma were associated with a favorable outcome, but the contribution of Rituximab in combined therapy was uncertain and the authors suggested that it warrants further investigation. ${ }^{11}$

We evaluated the effect of combining Rituximab individually with three different chemotherapeutic drugs commonly used in therapy and research and looked for additive or synergistic effects. We selected these drugs because they have distinct mechanisms of action. Hydroxyurea reduces the proliferative capacity of cells and induces senescence-like changes, and eventually causes cells to arrest at G1. ${ }^{12-15}$ Etoposide in vitro arrests Burkitt's lymphoma cell lines ${ }^{14}$ and other cells ${ }^{15}$ in S/G2 by blocking DNA replication. In contrast, vincristine disrupts cellular microtubules and prevents the formation of a functional spindle, resulting in the accumulation of cells in the mitotic phase. ${ }^{8}$

In vitro, pretreatment of a B lymphoma line with Rituximab sensitizes them to cytotoxic drugs, ${ }^{16}$ and some clinical trials have shown that pretreatment with Rituximab before each chemotherapy results in an additive effect. ${ }^{5}$ However, improved effects have also been achieved in vitro by simultaneous application of Rituximab and chemotherapeutic agents. ${ }^{10}$ We observed synergistic effects when CD20-expressing

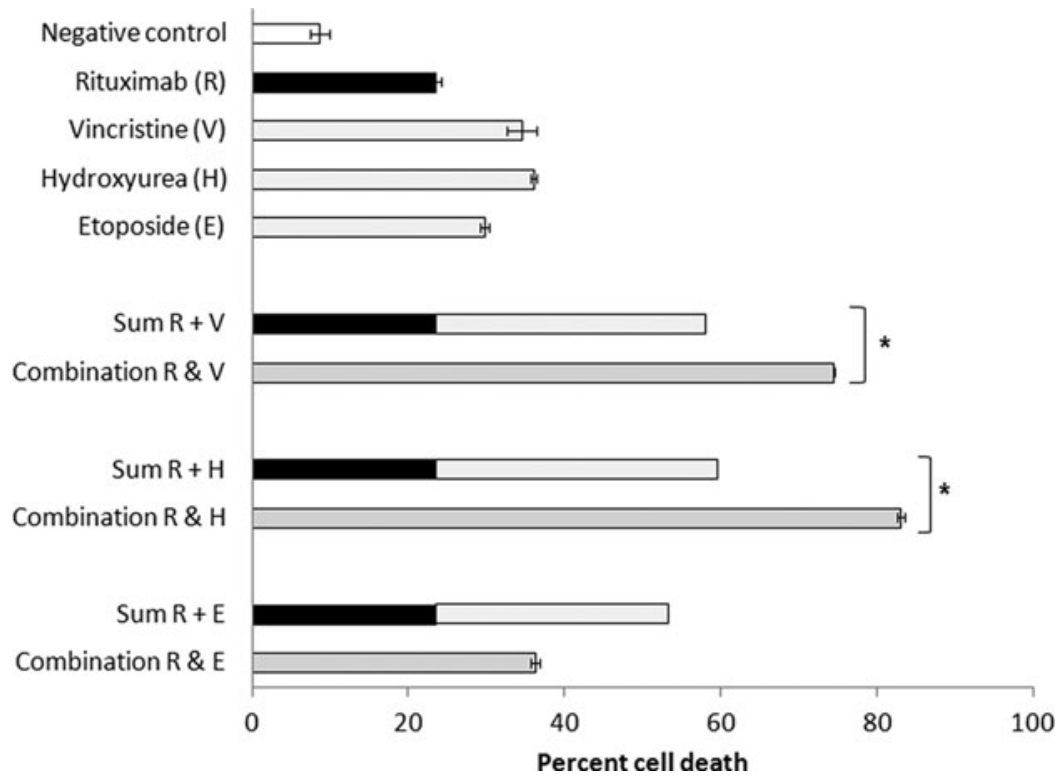

FIG. 2. Percent total cell death induced in Ramos Burkitt's lymphoma B cell line by Rituximab, hydroxyurea, vincristine, etoposide, and combinations thereof. Cells were incubated for 48 hours with various treatments: Rituximab $10 \mu \mathrm{g} / \mathrm{mL}$, hydroxyurea $1 \mathrm{mM}$, vincristine $2 \mu \mathrm{M}$, and etoposide $10 \mu \mathrm{M}$. "Sum" refers to the mathematical sum of individual treatments. Values are means $(n=3)$ and error bars are standard deviations. Asterisks: effect of combined treatment is significantly higher $(p<0.001)$ than mathematical sum of corresponding individual effects. 
human Burkitt's lymphoma cells (Ramos) were incubated simultaneously with Rituximab in combination with either vincristine or hydroxyurea. This synergy seems to be independent of the mode of action of the chemotherapeutic agents, as vincristine and hydroxyurea have different mechanisms of action. This finding might have clinical relevance, particularly in cases of Burkitt's lymphoma, where Rituximab is not significantly associated with improved overall survival. It is worthwhile to evaluate the effect of combining Rituximab individually with other chemotherapeutics, and depending on the results, it can also be tested with pairs of chemotherapeutics. We conclude that in vitro studies could lay the ground for developing immunochemotherapeutic treatments with fewer agents than currently used treatments and hence with fewer side-effects.

\section{Acknowledgment}

This work was funded by the Libyan Authority for Research, Science, and Technology, Tripoli, Libya.

\section{Disclosure Statement}

The authors declare no conflict of interest and no conflict between them.

\section{References}

1. Plosker GL, Figgitt DP. Rituximab: A review of its use in non-Hodgkin's lymphoma and chronic lymphocytic leukaemia. Drugs 2003;63:803.

2. Marcus R, Hagenbeek A. The therapeutic use of rituximab in non-Hodgkin's lymphoma. Eur J Haematol Suppl 2007;67:5.

3. van Meerten T, Hagenbeek A. CD20-targeted therapy: The next generation of antibodies. Semin Hematol 2010;47:199.

4. Rambaldi A, Lazzari M, Manzoni C, et al. Monitoring of minimal residual disease after $\mathrm{CHOP}$ and rituximab in previously untreated patients with follicular lymphoma. Blood 2002;99:856.

5. Czuczman MS, Grillo-Lopez AJ, White CA, et al. Treatment of patients with low-grade B-cell lymphoma with the combination of chimeric anti-CD20 monoclonal antibody and CHOP chemotherapy. J Clin Oncol 1999;17:268.
6. Vose JM, Link BK, Grossbard ML, et al. Long-term update of a phase II study of rituximab in combination with $\mathrm{CHOP}$ chemotherapy in patients with previously untreated, aggressive non-Hodgkin's lymphoma. Leuk Lymphoma 2005;46:1569.

7. Elledge SJ, Zhou Z, Allen JB. Ribonucleotide reductase: Regulation, regulation, regulation. Trends Biochem Sci 1992;17:119.

8. Jordan MA, Wilson L. Microtubules as a target for anticancer drugs. Nat Rev Cancer 2004;4:253.

9. Hochhauser D, Harris AL. The role of topoisomerase II alpha and beta in drug resistance. Cancer Treat Rev 1993;19:181.

10. Ghetie MA, Bright H, Vitetta ES. Homodimers but not monomers of Rituxan (chimeric anti-CD20) induce apoptosis in human B-lymphoma cells and synergize with a chemotherapeutic agent and an immunotoxin. Blood 2001;97: 1392.

11. Wasterlid T, Brown PN, Hagberg $\mathrm{O}$, et al. Impact of chemotherapy regimen and rituximab in adult Burkitt lymphoma: A retrospective population-based study from the Nordic Lymphoma Group. Ann Oncol 2013;24:1879.

12. Ajchenbaum F, Ando K, DeCaprio JA, et al. Independent regulation of human D-type cyclin gene expression during G1 phase in primary human T lymphocytes. J Biol Chem 1993;268:4113.

13. Hayashi N, Tsutsui T. Cell cycle dependence of cytotoxicity and clastogenicity induced by treatment of synchronized human diploid fibroblasts with sodium fluoride. Mutat Res 1993;290:293.

14. Lin CK, Nguyen TT, Morgan TL, et al. Apoptosis may be either suppressed or enhanced with strategic combinations of antineoplastic drugs or anti-IgM. Exp Cell Res 1998; 244:1.

15. Pocsik E, Mihalik R, Penzes M, et al. Effect of cell cycle on the regulation of the cell surface and secreted forms of type I and type II human tumor necrosis factor receptors. J Cell Biochem 1995;59:303.

16. Demidem A, Lam T, Alas S, Hariharan K, Hanna N, Bonavida B. Chimeric anti-CD20 (IDEC-C2B8) monoclonal antibody sensitizes a B cell lymphoma cell line to cell killing by cytotoxic drugs. Cancer Biother Radiopharm 1997;12: 177. 